\title{
Isolation of Pigmentation Mutants of Pseudomonas phenazinium
}

\author{
By G. S. BYNG AND J. M. TURNER \\ Department of Biochemistry, University of Liverpool, P.O. Box I47, \\ Liverpool L69 $3 B X$
}

(Received I2 April I976; revised 9 June 1976)

SUMMARY

Pigmentation mutants of Pseudomonas phenazinium unable to synthesize iodinin, or producing it only in reduced amounts, were isolated. The abilities of the mutants to synthesize nine other phenazines were also altered. Cross-feeding experiments and the altered patterns of pigment production suggested metabolic relationships between the phenazine pigments, and a scheme for their biosynthesis is proposed.

\section{INTRODUCTION}

Pseudomonas phenazinium synthesizes the water-insoluble phenazine pigment iodinin, first identified as I,6-dihydroxyphenazine 5,10-dioxide by Clemo \& Daglish (1950), when grown on a variety of carbon sources, the highest yield being obtained on L-threonine (Bell \& Turner, 1973). The pseudomonad also elaborates a number of relatively water-soluble pigments (Bell \& Turner, I973), the identities of which have been established (Byng \& Turner, 1975). In all, Io distinct phenazines have been recognized.

Over 30 phenazines occur naturally, all produced by bacteria, but in no case is the route of biosynthesis known. Although shikimic acid acts as a biosynthetic precursor in many such cases, for example in the synthesis of iodinin and pyocyanine, and chorismic acid is the branch-point compound leading to pyocyanine in Pseudomonas aeruginosa (Calhoun, Carson \& Jensen, 1972; Longley et al., 1972), little further information on their biosynthesis is available. A procedure for isolating pyocyanine mutants in $P$. aeruginosa has been described (Carson \& Jensen, 1974), but no details of the altered patterns of pigment production by such pigment phenotypes have been described.

We report here the isolation of iodinin mutants of $P$. phenazinium. The phenazine pigments produced by a number of the mutants have been examined_and cross-feeding experiments have indicated metabolic relationships.

\section{METHODS}

Bacteria. Pseudomonas phenazinium, isolated by Bell \& Turner (1973), has been deposited with the National Collection of Industrial Bacteria, accession number NCIBII027. The mutants described are available from the authors.

Growth of bacteria. The minimal growth medium contained $\left(\mathrm{g} \mathrm{l}^{-1}\right): \mathrm{KH}_{2} \mathrm{PO}_{4}, 8 \cdot 43 \mathrm{~g}$; $\mathrm{Na}_{2} \mathrm{SO}_{4}$ (anhydrous), I.2 g; $\mathrm{MgSO}_{4} \cdot 7 \mathrm{H}_{2} \mathrm{O}, 0 . \mathrm{I} \mathrm{g}$; L-threonine, $2 \mathrm{~g}$; adjusted to $\mathrm{pH} 5.5$. Liquid cultures were incubated in a shaker-incubator at $30^{\circ} \mathrm{C}$. Plates containing the same medium solidified with $2 \%(\mathrm{w} / \mathrm{v})$ agar were also incubated at $30^{\circ} \mathrm{C}$.

Mutagenesis. The procedure was based on that described for mutants of a pseudomonad by Heptinstall \& Quayle (I970) as modified by A. L. Herrera (unpublished). A culture of the 
wild-type organism was grown on minimal medium, harvested, washed once with sterile buffer (Tris/maleate; $\mathrm{pH} 6.0 ; 50 \mathrm{~mm}$ ) and resuspended in the same buffer. To $1.0 \mathrm{ml}$ portions of the suspension was added an equal volume of a solution of $N$-methyl- $N^{\prime}$-nitro-

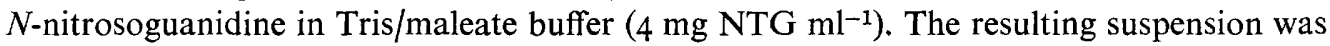
incubated with shaking at $30^{\circ} \mathrm{C}$ for $\mathrm{I} 5 \mathrm{~min}$. The bacteria were then harvested by centrifuging, washed twice with sterile water and resuspended in sterile minimal medium. The suspension was serially diluted and $0.1 \mathrm{ml}$ samples were spread on plates to give about 100 colonies per plate. Plates were incubated for about 4 days at $30^{\circ} \mathrm{C}$ before being examined for pigmentation mutants. Putative mutants, usually distinguished by the absence of iodinin from colonies, were picked off and streaked on to new plates.

Extraction and examination of phenazines. At the appropriate phase of growth the liquid cultures were adjusted to $\mathrm{pH} 2$ by adding conc. $\mathrm{HCl}$. An equal volume of chloroform was added to the acidified culture, and the pigments were extracted by shaking the mixture overnight at $30^{\circ} \mathrm{C}$. Usually a second extraction, with an equal volume of $\mathrm{CHCl}_{3}$, was required to remove all traces of iodinin. The spectra of $\mathrm{CHCl}_{3}$ extracts were examined in the u.v. and visible wavelength region. Samples of the extracts were then vigorously shaken with equal volumes of aqueous $\mathrm{NaHCO}_{3}(5 \%, \mathrm{w} / \mathrm{v})$, which removes most phenazines other than iodinin, I,6-dihydroxyphenazine and its $\mathrm{N}$-monoxide, and the $\mathrm{CHCl}_{3}$ phases were re-examined spectroscopically.

Samples $(\mathrm{I} \cdot \mathrm{O} \mathrm{ml})$ of the original $\mathrm{CHCl}_{3}$ extracts were chromatographed by thin-layer chromatography (t.l.c.) on silica gel $\mathrm{G}$ (Merck) using $\mathrm{CHCl}_{3} /$ glacial acetic acid ( $9: \mathrm{I}$, by vol.), and examined visually after drying. Pigment bands, provisionally identified by their $R_{F}$ values (Byng \& Turner, I975), were eluted from the silica using appropriate solvents and examined by spectroscopic and other methods.

\section{RESULTS}

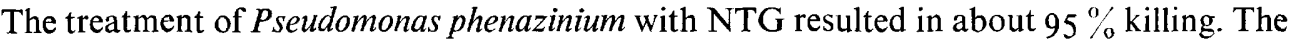
survivors were plated out for clonal isolation. Mutants with altered pigmentation were detected visually, the absence of iodinin from the morphologically compact colonies being the most obvious trait. The incidence of such mutants was I to $2 \%$ among the survivors of mutagenesis.

Individual pigmentation variants were examined by growth in liquid medium. Cultures in the stationary phase were acidified and pigments were extracted and examined. About $80 \%$ of the mutants failed to produce any phenazine in detectable amounts. Of the other mutants examined, many failed to produce iodinin in liquid or solid media, but some did produce the phenazine although in markedly reduced amounts compared with the wild type. The altered ability of mutants to synthesize iodinin was frequently compensated for by the production of other phenazines in amounts much greater than in the wild type. Mutants in which the predominant pigment was I,6-dihydroxyphenazine, I,6-dihydroxyphenazine 5-monoxide, 9-hydroxyphenazine I-carboxylic acid plus 2,9-dihydroxyphenazine I-carboxylic acid, and phenazine r,6-dicarboxylic acid, respectively, were isolated (Table I). The pattern of pigment production was altered in all mutants, and none of them produced only one phenazine.

Cross-feeding experiments demonstrated that iodinin can be synthesized from other phenazines. The mutant strain D34, producing predominantly but not exclusively I,6-dihydroxyphenazine, was streaked against all of the non-pigmented strains $I Z$ to $26 \mathrm{z}$. In each case iodinin was formed by the non-pigmented strains at the junction of the two streaks. 


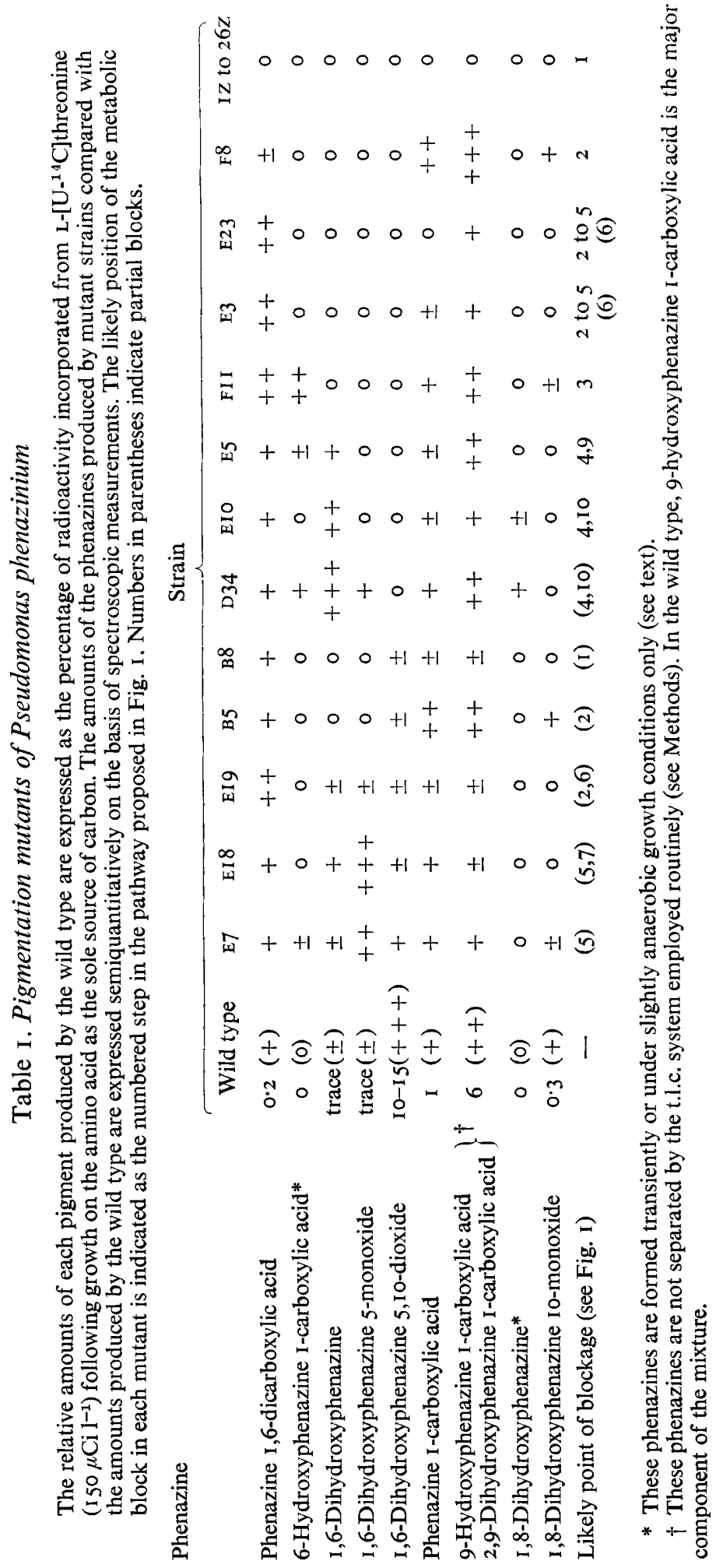




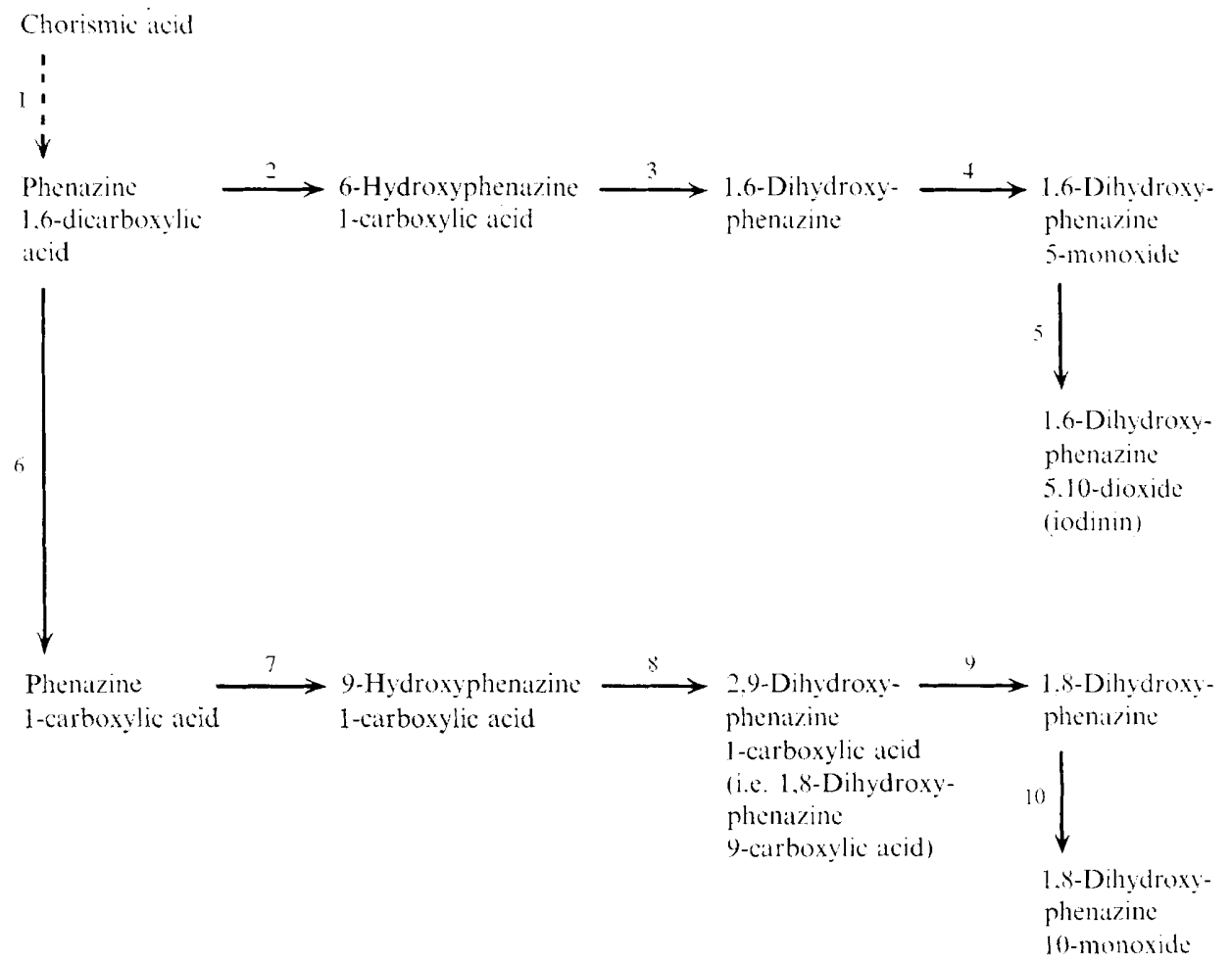

Fig. I. Postulated pathway for the biosynthesis of phenazine pigments by Pseudomonas phenazinium.

This showed that these strains were not contaminants; a variety of unrelated bacteria also capable of growth on L-threonine (Bell et al., 1972) did not produce iodinin when plated against strain D34. The best iodinin production occurred with strains $3 \mathrm{Z}, 4 \mathrm{Z}, 8 \mathrm{Z}, \mathrm{I}$ I Z, I3Z, $\mathrm{I} 7 \mathrm{Z}$ and $\mathrm{I} 8 \mathrm{Z}$. The feeding of chemically pure $\mathrm{r}, 6$-dihydroxyphenazine, added as a small crystal to the centre of freshly inoculated plates, resulted in iodinin formation by these seven non-pigmented strains. No iodinin was formed when these strains were tested against strains E3 and E23, neither of which excreted 1,6-dihydroxyphenazine.

Additional cross-feeding experiments between the strains listed in Table I, together with the altered patterns of pigment production, suggested further precursor-product relationships. These are incorporated in the scheme proposed for the biosynthesis of phenazine pigments (Fig. I). Thus, strain FII, which atypically excreted 6-hydroxyphenazine I-carboxylic acid but no 1,6-dihydroxyphenazine, promoted iodinin formation by the nonpigmented $z$ strains when streaked against them. When strains E3, E23, F8 and FI I, none of which produced I,6-dihydroxyphenazine, its 5-monoxide or iodinin, were cross-fed with the I,6-diol from strain D34, iodinin was produced by strains F8 and FI I only. It seemed likely that the metabolic block in strain $\mathrm{F} 8$ affected the formation of 6-hydroxyphenazine I-carboxylic acid (step 2, Fig. I) whereas its decarboxylation to I,6-dihydroxyphenazine (step 3, Fig. I) was blocked in strain FI I. The formation of iodinin by F8 when streaked against FI I confirmed that 6-hydroxyphenazine I-carboxylic acid was a precursor. The inability of E3 and E23 to form iodinin from either 6-hydroxyphenazine I-carboxylic acid or I,6-dihydroxyphenazine suggested that these mutants were multiply blocked (steps 2, 3,4 and probably 5, Fig. I). The probable positions of metabolic blocks in each mutant are indicated in Table I. 
Mutant strains E3, EI9 and E23 accumulated phenazine I,6-dicarboxylic acid but only trace amounts of some other phenazines. This suggested that the dicarboxylic acid was a common precursor, possibly a branch-point compound. The mutant $F 8$, in which the formation of iodinin and its precursors was blocked, was found to produce relatively large amounts of phenazine I-carboxylic acid and 9-hydroxyphenazine I-carboxylic acid plus 2,9-dihydroxyphenazine I-carboxylic acid (i.e. I,8-dihydroxyphenazine 9-carboxylic acid), which suggested that these compounds were metabolically related. I,8-Dihydroxyphenazine I0-monoxide was formed by $P$. phenazinium only after growth had ceased and appeared to be an endproduct. By analogy with the formation of 1,6-dihydroxyphenazine 5-monoxide (step 4, Fig. I), I,8-dihydroxyphenazine was a likely precursor of its I0-monoxide, and when conditions became anaerobic towards the end of growth the I,8-diol accumulated rather than the I o-monoxide. Mutants D34 and Ero appeared to be blocked at this point (step Io, Fig. I), as indicated in Table $\mathrm{I}$.

The non-pigmented $\mathrm{z}$ strains were of interest as possible producers of the immediate precursor of the first-formed phenazine. All $26 \mathrm{z}$ strains were streaked against each other. In no case did cross-feeding between non-pigmented strains result in pigment formation.

\section{DISCUSSION}

The availability of pigmentation mutants of $P$. phenazinium offers the opportunity to study the route of iodinin biosynthesis by classical procedures. Many of the strains listed in Table I are convenient sources of phenazines for purification and feeding to either wild type or mutant strains. The use of radioactive phenazines, obtained by administering $\left[{ }^{14} \mathrm{C}\right]$ shikimate under suitable conditions, would be an extension of this approach. Podojil \& Gerber (1967) have already shown that radioactive I,6-dihydroxyphenazine and its 5monoxide are efficient precursors of iodinin in Brevibacterium iodinum and Herbert, Holliman \& Ibberson (1972) have similarly demonstrated the metabolism of 6-hydroxyphenazine I-carboxylic acid to iodinin by the same bacterium. These results are consistent with the scheme proposed for the biosynthesis of phenazines (Fig. I) on the basis of the cross-feeding experiments with pigmentation mutants.

The evidence for phenazine I,6-dicarboxylic acid acting as a branch-point compound, and for the pathway leading to I,8-dihydroxyphenazine I0-monoxide (Fig. I) is incomplete but is consistent with the results. Decarboxylation, hydroxylative decarboxylation and hydroxylation reactions are well known. Even so, the involvement of phenazine 1,6-dicarboxylic acid is proposed solely on the basis of its accumulation by some mutant strains. The failure of the dicarboxylic acid to act as a precursor of iodinin, observed in the cross-feeding experiments and also reported by Herbert et al. (1972), may be due to permeability factors. In support of the role for phenazine I-carboxylic acid indicated in Fig. $I$, the feeding of the $\left[{ }^{14} \mathrm{C}\right]$-labelled compound to growing cultures of $P$. phenazinium resulted in the formation of radioactive 9-hydroxyphenazine I-carboxylic acid and 2,9-dihydroxyphenazine I-carboxylic acid. In turn, the feeding of these $\left[{ }^{14} \mathrm{C}\right]$-labelled compounds gave rise to radioactive $\mathrm{I}, 8$ dihydroxyphenazine Io-monoxide (L. Swift and J. M. Turner, unpublished results).

The high incidence of non-pigmented strains suggests that the number of steps between chorismic acid and the hypothetical key intermediate common to all natural phenazines (Podojil \& Gerber, 1970) may be large. It should be noted, however, that the mutant selection procedure failed to detect pigment phenotypes other than those in which the production of iodinin itself was affected. The non-pigmented strains are likely to form a heterogeneous group. Factors responsible for the initiation and termination of secondary metabolite pro- 
duction are complex (Weinberg, I970; Demain, 1973) and it is likely that the lesion in many mutants does not directly involve the route from chorismic acid. Even so it is surprising that no pigment formation occurred as a result of cross-feeding between the large number of non-pigmented strains tested. Again, impermeability of the cell membrane may be responsible or, perhaps, the lability of intermediates between chorismate and phenazines.

This work was carried out with the aid of a grant from the Medical Research Council. The interest and support of Professor T. W. Goodwin, F.R.S., is gratefully acknowledged.

\section{REFERENCES}

Bell, S. C. \& Turner, J. M. (1973). Iodinin biosynthesis by a pseudomonad. Biochemical Society Transactions I, 75I-753.

Bell, S. C., Turner, J. M., Collins, J. \& Gray, T. R. G. (I972). Patterns of microbial threonine catabolism: a survey. Biochemical Journal 127, $77 \mathrm{P}$.

Byng, G. S. \& Turner, J. M. (I975). Phenazine biosynthesis by a pseudomonad. Biochemical Society Transactions 3, 742-744.

Calmoun, D. H., Carson, M. \& Jensen, R. A. (1972). The branch point metabolite for pyocyanine biosynthesis in Pseudomonas aeruginosa. Journal of General Microbiology 72, 58I-583.

Carson, M. \& Jensen, R. A. (1974). Phenotypic recognition of pyocyanine mutants in Pseudomonas aeruginosa. Journal of Bacteriology I17, 312-3I4.

Clemo, G. R. \& Daglish, A. F. (I950). The phenazine series. VIII. The constitution of the pigment of Chromobacterium iodinum. Journal of the Chemical Society, 1481-1485.

Demain, A. L. (1973). Mutation and the production of secondary metabolites. Advances in Applied Microbiology 16, 177-202.

Heptinstall, J. \& Quayle, J. R. (1970). Pathways leading to and from serine during growth of Pseudomonas AMI on $C_{1}$ compounds or succinate. Biochemical Journal $\mathbf{1 1 7}, 563-572$.

Herbert, R. B., Holliman, F. G. \& IbBerson, P. N. (I972). Incorporation of 6-hydroxyphenazine I-carboxylic acid into iodinin. Journal of the Chemical Society, Chemical Communications, 355-356.

Longley, R. P., Halliwell, J. E., Campbell, J. J. R. \& Ingledew, W. M. (1972). The branchpoint of pyocyanine biosynthesis. Canadian Journal of Microbiology 18, I357-I363.

PodoJil, M. \& Gerber, N. N. (1967). The biosynthesis of 1,6-phenazinediol 5,10-dioxide (iodinin) by Brevibacterium iodinum. Biochemistry, New York 6, 2701-2705.

PodoJil, M. \& Gerber, N. N. (1970). Biosynthesis of 1,6-phenazinediol 5,10-dioxide (iodinin). Incorporation of shikimic acid. Biochemistry, New York 9, 46I6-4618.

WeINBERG, E. D. (1970). Biosynthesis of secondary metabolites: roles of trace metals. Advances in Microbial Physiology 4, I-44. 